\title{
Inconsistent Normalized Intensities for Quantitative STEM: Detector Scans and Single Electron Counting
}

\author{
Xiahan Sang ${ }^{1}$, James M. LeBeau ${ }^{1}$ \\ 1. Department of Materials Science \& Engineering, North Carolina State University, Raleigh, NC 27606
}

STEM images placed on an absolute scale relative to the incident beam intensity have helped solve numerous materials science problems. Two normalization methods have been proposed: the detector scan (DS) method [1], which measures the incident beam intensity directly on the STEM detector, and the single electron count (SEC) method that uses the signal for a single electron and separately measured beam current to determine absolute intensities [2]. In this talk, we will demonstrate that a drastic difference in normalization can occur between the two methods as shown in Figure 1a. For a fair comparison, two major error sources need to be taken into consideration. First, the HAADF detector experiences extremely low beam current $\left(<0.1 \mathrm{e}^{-} / \mu \mathrm{s}\right)$ in the SEC method compared with the DS approach $\left(>10 \mathrm{e}^{-} / \mu \mathrm{s}\right)$. As a result, detector non-linearity can play an important role. Second, the detector non-uniformity, which is known to cause 10\%-20\% error, must be taken into account for both methods.

We explore the cause of the DS/SEC difference by imaging the HAADF with very low beam current $\left(0.073 \mathrm{e}^{-} / \mu \mathrm{s}\right.$, measured using a calibrated CCD). For short pixel dwell times (Figure $\left.2 \mathrm{a}, 16 \mu \mathrm{s}\right)$, the detector response yields discrete events, with only one or two electrons hitting the detector. Because the FEI hardware/software averages the detector signal, the single electron intensity is inversely related to the dwell time. As an example, at $16 \mu \mathrm{s}$ the single electron intensity corresponds to 755 arb. units as measured by the difference between the first peak and the background. At intermediate dwell times (Figure 2b), three discrete peaks are observed sitting on a broad peak that corresponds to pseudocontinuous illumination during the well probe dwell time. For very large dwell time (Figure 2c, $256 \mu$ s), only the pseudo-continuous broad peak is observed and represents the incident beam intensity, or 628 arb. units.

As the influence of detector non-linearity and non-uniformity are incorporated, the results of the SE detector maps can be directly compared with the DS method. From the SEC approach, the apparent beam current is $0.052 \mathrm{e}^{-} / \mu \mathrm{s}$, which is the ratio of the beam intensity (628) from $256 \mu$ s detector image and product of the single electron intensity (47.2, scaled from $16 \mu \mathrm{s})$ and dwell time $256 \mu$ s. Compared with the CCD measured beam current $\left(0.073 \mathrm{e}^{-} / \mu \mathrm{s}\right)$, a correction factor of 1.4 is required to account for the intensity discrepancy. Thus, under these imaging conditions, the apparent single electron signal does not capture the true intensity of a single electron. After accounting for the mismatch, for example, we achieve significantly better agreement between SEC and DS methods (red vs. black in Figure 1b). Finally, we will discuss other sources of error that contribute to the correction factor, including the detector electronics and control software that can further improve the method agreement [3]. 


\section{References:}

[1] J. M. LeBeau and S. Stemmer, Ultramicroscopy 108 (2008), p. 1653.

[2] R. Ishikawa et al., Microscopy and Microanalysis 20 (2014), p. 176.

[3] The authors acknowledge the use and support of the Analytical Instrumentation Facility at North Carolina State University, which is supported by the State of North Carolina and the National Science Foundation.

(a)

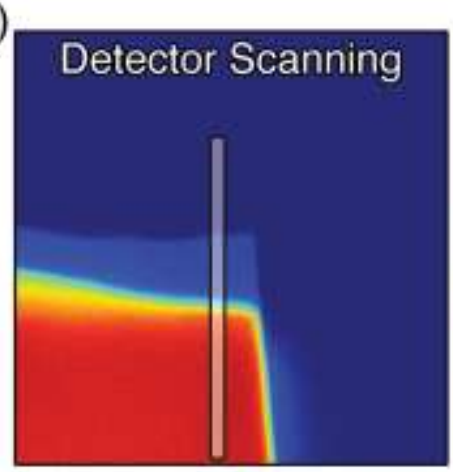

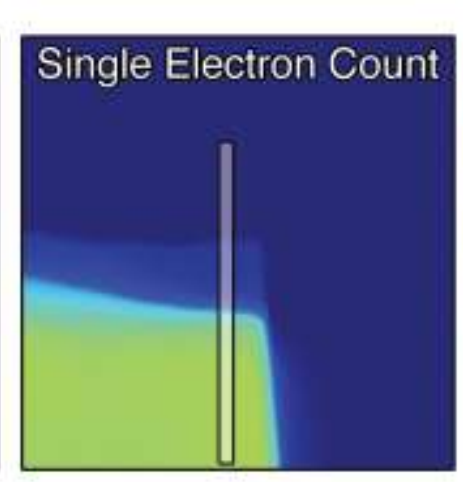
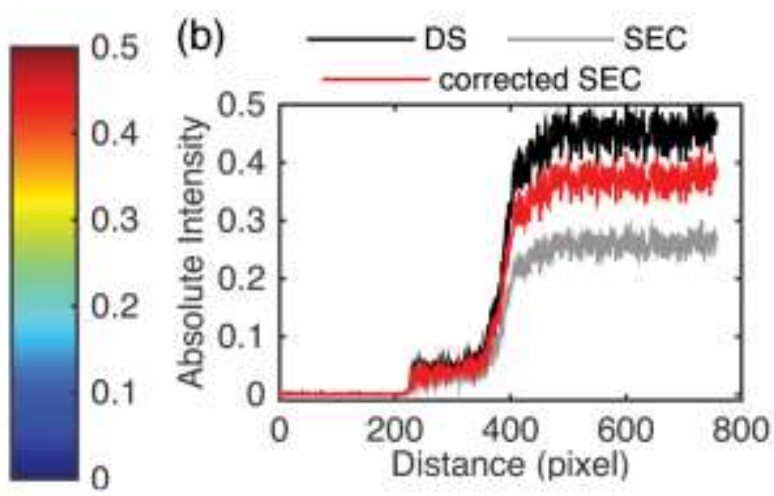

Figure 1. (a) Normalized intensity of a STEM image using two different methods: detector scanning and single electron count. (b) Line profiles extracted from the translucent white lines from each map.

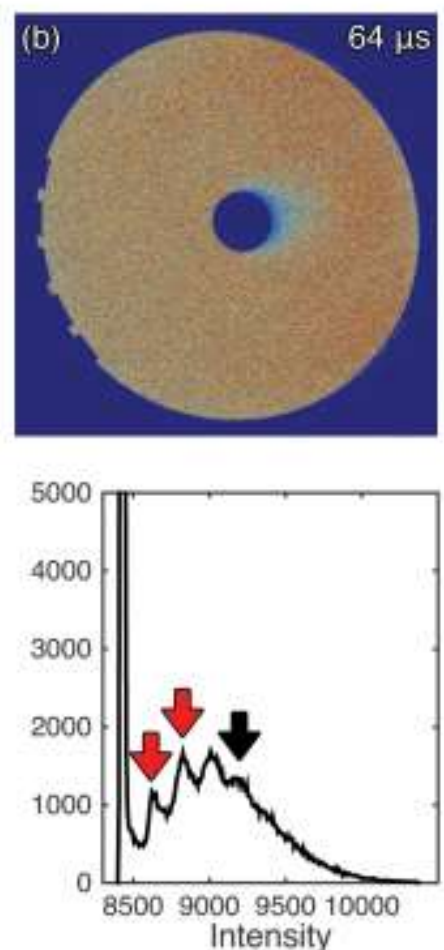
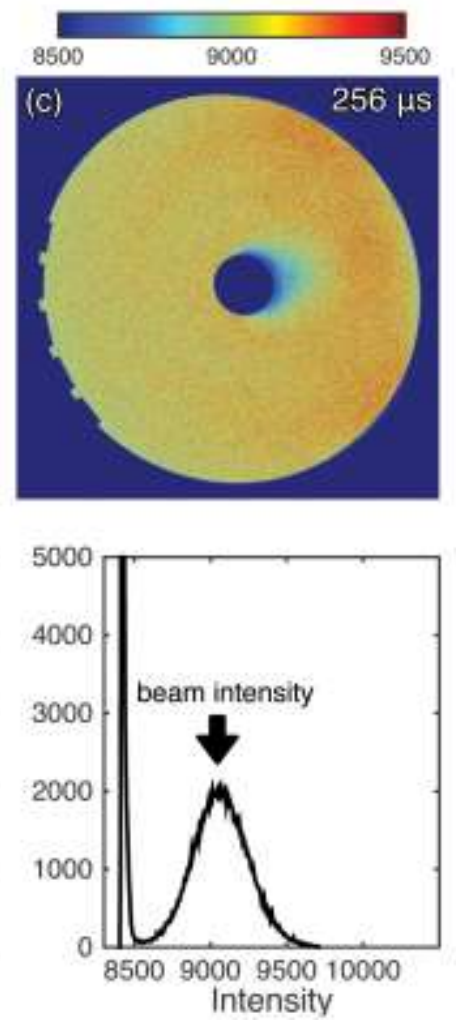

Figure 2. Detector mapping and corresponding histogram with different dwell times: (a) $16 \mu \mathrm{s}$, (b) 64 $\mu \mathrm{s}$, (c) $256 \mu \mathrm{s}$. The red arrows correspond to discrete electron counts while the black arrows correspond to the incident beam intensity. 